\title{
How Machine Learning Potentials are transforming the Practice of Digital Marketing: State of the Art
}

\author{
Kenza Bayoude $^{1}$, Youssef Ouassit ${ }^{2}$, Soufiane Ardchir ${ }^{3}$, Mohamed Azouazi ${ }^{4}$ \\ 1,2,3,4 Departement of mathematics and computer science, Faculty of Sciences, Hassan II University, Casablanca, Morocco
}

\section{Article Info}

Received Jun 25, 2018

\section{Keyword:}

Machine Learning

Digital Marketing

Predictive Analysis

Customer Behavior

\begin{abstract}
Today, the digital marketing is constantly evolving, new tools are regularly introduced with the new consumer habits and the multiplication of data, often forcing marketers to delve into too much data that may not even give them the overview they need to make business decisions that have an impact.

After the revolution of machine learning technology in other real world application, machine learning is changing the digital marketing landscape, $84 \%$ of marketing organizations are implementing or expanding their use of machine learning in 2018[1].It becomes easier to predict and analyze consumer behavior with great accuracy.

In our work we will start by establish an art of state on the main and most used machine learning potentials in digital marketing strategies and we show how machine learning tools can be used at large scale for marketing purposes by analyzing extremely large sets of data. The way that ML is integrated in digital marketing practices helps them better understand the target consumers and optimize their interactions with them.
\end{abstract}

\section{Corresponding Author:}

Kenza Bayoude,

$\mathrm{PhD}$ Student Departement of mathematics and computer science,

Hassan II University,Casablanca,Morocco

Email: kenza.bayoude@gmail.com

\section{Introduction}

It is In recent years, the marketing field has been impacted by advanced technologies; it is changing rapidly with advancements being made in machine learning coupled with increases in the availability and quantity of data, more companies are looking in to adapt the concept of machine learning in their marketing strategies to make their operations more effective and smarter. According to QuanticMind, 97\% of executives estimate that the future of marketing rely on how digital marketers use machine learning potentials [2].

Increasingly, the marketing world is using these resources to understand data, and learn more about customers, and streamline operations marketing. Marketers leverage the ability of machine learning to connect data points to gain insight into their customers and process critical data to predict the future.

Our research is a descriptive study, it aims to present the various dimensions attached to the concept of machine learning in marketing to improve the marketing strategies, and we explain how machine learning tools are incorporating in the digital marketing strategies.

Through this paper, we propose a state of the art of the transformative ways in which machine learning technology is changing the world of digital marketing, we begin with basic concepts on machine learning then we present its role in business world. The last part is reserved to explore how the power of machine learning are utilizing to revolution a digital marketing and discusses the necessity of using this strategy for the marketing of product and services. 


\section{Machine learning: Concepts}

\subsection{Machine learning: Definition}

Machine learning is a branch of study that allows computers the ability to learn and improve themselves by learning from data experience without basing on explicit programming, relied on the idea that computer systems can identify models and make decisions with minimal human intervention [3]. They can self-rulingly change. They gain from past counts to make strong, repeatable decisions and results. It's a science that isn't new but instead one that has expanded new power. While many machine learning computations have been around for a long time, the ability to normally apply complex numerical [15].

Within the field of predictive analytics, machine learning is a method of data analysis that improves and automates analytical model building. It's used to develop complex predictive algorithms and to generate more precise mathematical models that analyze larger amounts of increasingly current and historical data to make predictions about future events. The adoption of data intensive machine learning methods can be found throughout technology, science and ecommerce, which leads to evidence-based decision making in many domains, including marketing and other sectors [4].

\subsection{Machine learning: The Process}

Machine Learning consists of series of steps to be followed.

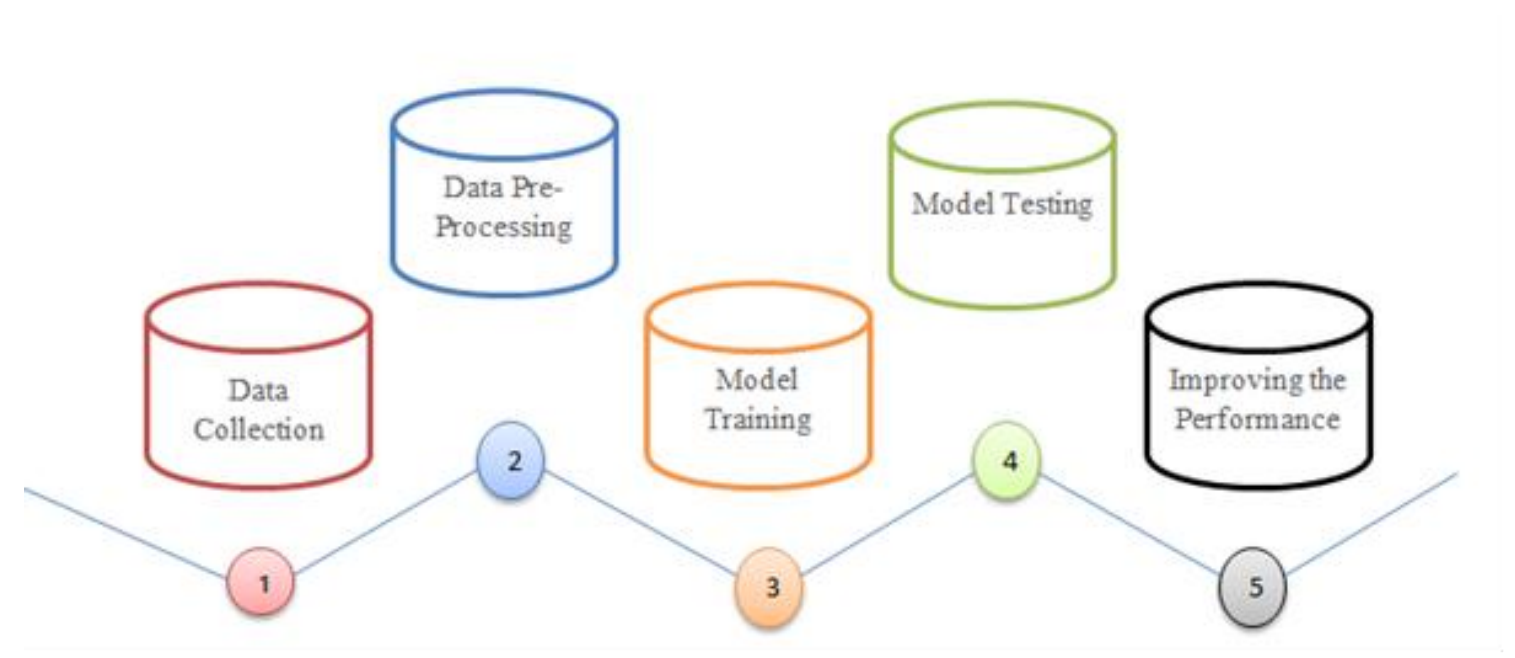

Figure1.Machine learning process.

\subsubsection{Data collection}

The first step and most importantly is to gather relevant data according to the problem statement.

\subsubsection{Data Pre-processing}

In this process, the raw data is collected and you analyze the data to find a way to transform it into useful data.

\subsubsection{Model Training}

This training model requires providing a Machine learning algorithm with training data to be studied.

\subsubsection{Model Testing}

It is important to be able to evaluate the performance of the model and compare different algorithms to estimate the properties of model.

Because of the process of training and testing, data is often divided into two different subsets, training datasets and testing datasets. The main reason for this is to ensure that the model can generalize enough to make good predictions on new examples [5]. 


\subsubsection{Improving the performance}

The performance of the model can be improved both on the training and testing data sets by trying several machine learning algorithms and using the one that does the best.

\section{Role of Machine learning in Business}

Machine Learning is a topic of considerable interest in digital world and industry discussions and the advertising world these days.

Many companies use machine learning algorithms to highlight hidden knowledge in consumer data and to improve business, provide for better customer experience, and contribute to an operational efficiency like a speed, cost savings, and greater precision.

A variety of technological developments in the marketing sector have contributed to generate the infrastructure and data sets and the business organization gains a suitable solution to develop business processes, improve company performance, increase profits, investigate competitors' motive and activities and obtain additional satisfied customers[16].

The proliferation of electronic trading platforms has been accompanied by an augmentation in the disponibility of high quality market data in structured formats. In some countries, such as the United States, market regulators allow to use social media for public announcements [6].

In addition to making digitized data available for machine learning, the computerization of markets has made it feasible for machine learning algorithms to interact directly with markets, putting in realtime complex purchase and sell orders relied on sophisticated decision-making, in many cases with a little human intervention.Fig.2.

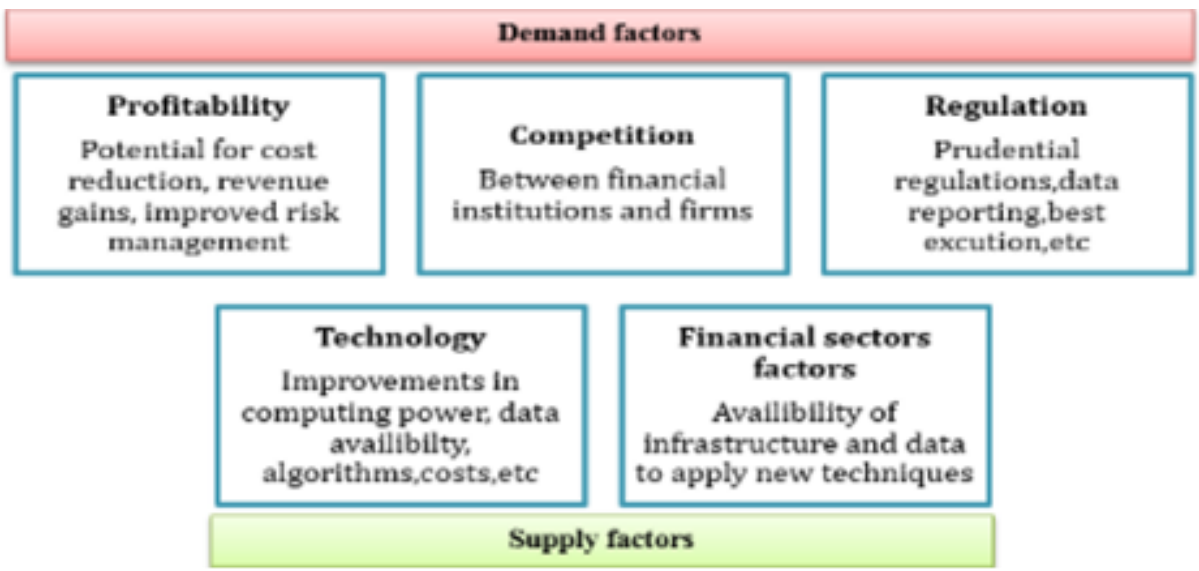

Figure 2. Demand and supply factors of marketing adoption of machine learning.

\section{The relationship between Machine learning and Digital Marketing}

Most companies are taking advantage of Machine Learning techniques because of having the capacity to analyze a large number of data sets and give understandable analysis that help marketers to specialize in other field to optimize the marketing strategies.

Machine Learning will define how digital marketing will be conducted now and in the future. The following are the ways that has transformed Machine Learning potentials in changing the world of digital marketing.

\subsection{Predicting the behavior of customers:}

Using Propensity models and predictive analytics, marketers can identify potential customers who are more likely to respond to their offers by correlating customer characteristics with their anticipated behaviors.

\subsubsection{Predictive analytics}


Predictive analysis is the analysis of available historical and current data and the use of machine learning techniques to create forecasts of future behaviors, preferences and needs. It aims to predict future trends, particularly in the marketing sector [7].

Predictive analysis allows marketers to extract information from data, that users leave when they interact online, and uses it to predict purchasing trends and user behavior models in order to develop automated systems and customer profiles to target certain markets.

This tool analyzes data in large volumes and helps reveal the most impactful insights. This works through the following steps [8]:

Identify the objectives that analyze data extracted from several sources, and that require cleaning and preparation to determine the pattern or model that suits our needs.

Creation of models and their validation. The models are validated based on the goals set.

Application of the model results to take advantages of it and guide to making the correct business decisions and continually refining the model for better results.

For businesses today, the key to optimize marketing campaigns is being able to anticipate customer behavior.

Predictive models generated by machine learning can be trained to the prospect list and make it possible to predict the ideal customer profiles based on relevant data from the web and certain criteria defined by the sales team as "qualified buyers." In this way, they can a predict lot of aspects, such as which clients are more vulnerable to making more than one purchase. This, in addition to contributing to raise the sales, means saving considerable time and resources. This is the essence of the machine learning workflow, which stores the relationships and applies them to make the prediction [9].

The model is trained with new data as it becomes available, which improves the reliability of future predictions.Fig.3.

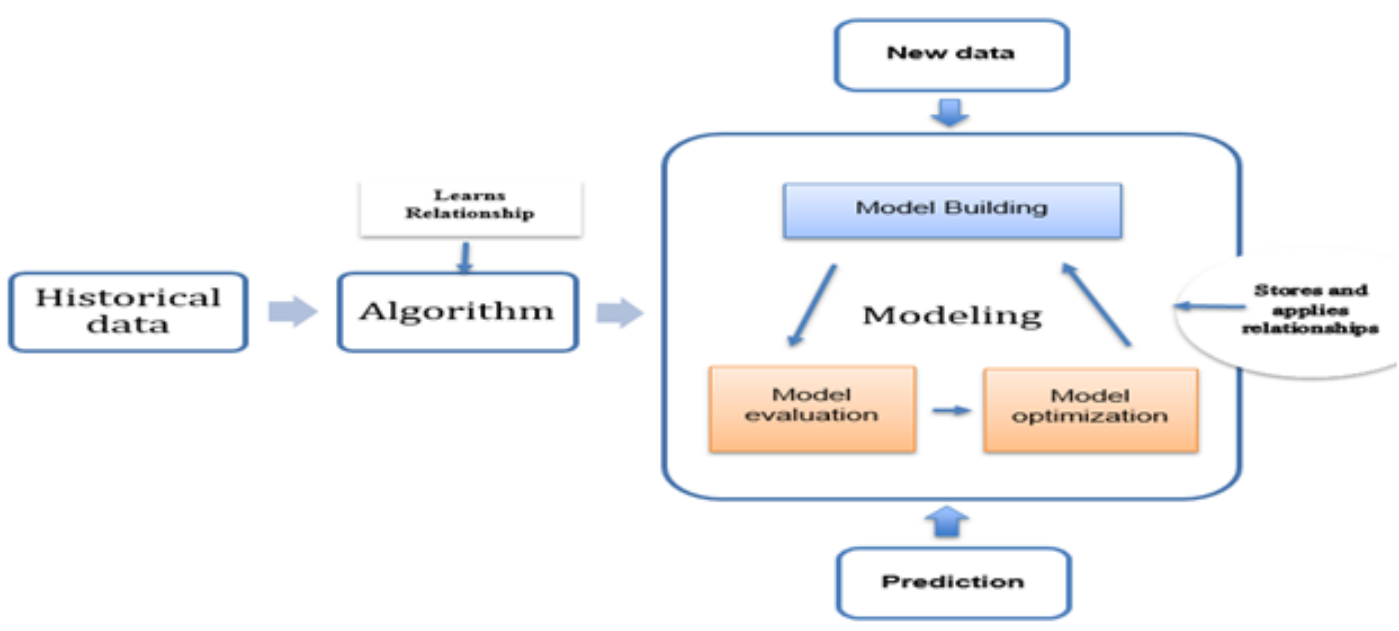

Figure 3. The components and processes of a machine learning model to predict a potential customers.

\subsubsection{Propensity Modeling}

A propensity model is statistical method to make accurate predictions about customer behavior; the creation of the model based on the data history powered the machine learning algorithm. Common models include predictive lifetime value; likelihood of engagement; propensity of unsubscribe; propensity of conversion; propensity of purchasing; and propensity of churn [10]. The simple diagram below shows the steps of this process: Fig. 4 


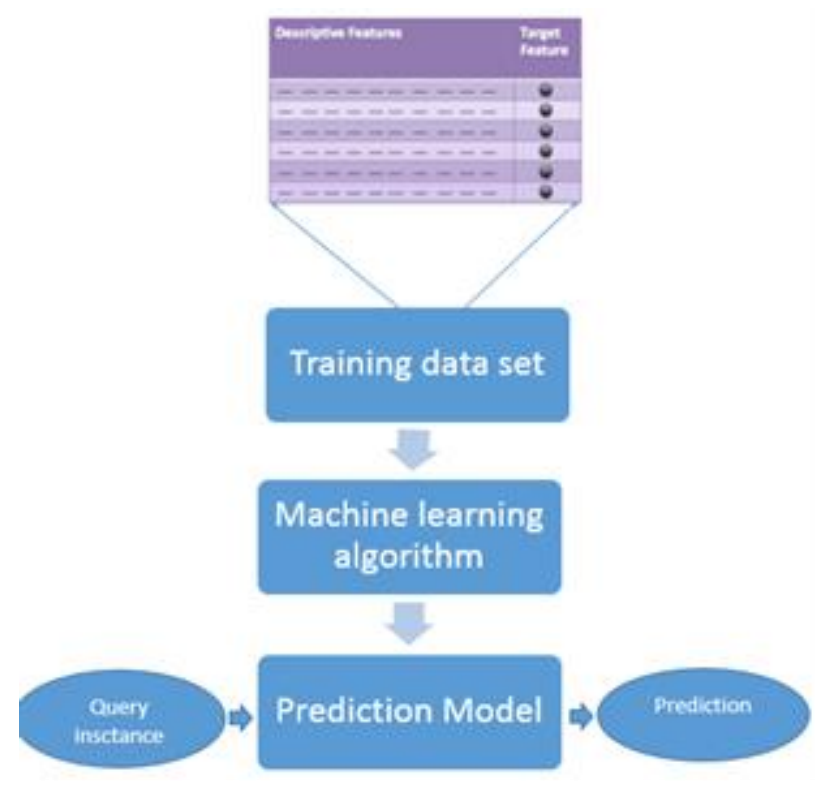

Figure 4 . The process of propensity modeling.

\subsection{Predictive Marketing}

Social media plays an important role in collecting more personal information about the potential clients, which makes it easier for marketers to have focused campaigns. With each click every time a user browses the internet, new data is generated and compiled for predictive analysis [12]. This data is valuable for marketers to optimize information and provide the most appropriate information.

\subsection{Chatbots}

Most businesses already know and use live chatbots. In short, Chatbots are driven programs that interact with users in natural language environments. This makes this website unique, valuable, or interesting and different from the others.

Most digital marketers see chatbots as a tool to provide customized customer service by gathering data and personal information. However, chatbots also can guide users through the customer's journey to sales [13].

From a digital marketing perspective, chatbots provide the possibility to engage with an audience that is targeted at a personal level.

\subsection{Content Marketing}

Nowadays, companies are embarking on content personalization and consumers are increasingly interested in interacting with them on an individual basis [14]. Thanks to machine learning tools, companies can now personalize customer experiences even if their customer profiles are very specific. They use also the power of Social Networks; it plays a communication media in real time for the user's interaction. They are used to share all the experiences and their personal valid opinions on various topics like news, politics, celebrities, sports, events and products.It exhibits high potential indigital marketing for integral growth [17].

Machine learning helps companies create a personalized content based on the consumer's profile, their expectations, behavior, buying habits and interests, companies can implement specific tools on their site such as algorithms that analyze data and chatbots. It is also possible to direct the Internet user to specific content thanks to personalized recommendation systems. 


\section{Conclusion}

Technological advancements have always helped businesses by creating new opportunities for reaching customers. One of greatest technologies of our time is machine learning. It creates new opportunities for storytelling and marketing which is change how people interact with information, technology, brands and services.

In this article we presented the state of the art of how machine learning techniques can improve the digital marketing practices, our next article we will in more detail about how applied propensity modeling to open new customer opportunities by gathering the data from social media.

\section{References}

[1] "Impact of Machine Learning on Marketing",https://satoristudio.net/machine-learning-in-digitalmarketing, 2019

[2] "How to Apply Machine Learning to Your Digital Marketing Strategy", Available on:

https://digitalmarketinginstitute.com/en-ie/blog/14-03-2018-how-to-apply-machine-learning-to-your-digitalmarketing-strategy,2019

[3] Alescodata "machine learning for direct mail marketing", 2018

http://www.alescodata.com/ebook/Alesco_Machine_Learning_Ebook.pdf

[4] M. I. Jordan1, T. M. Mitchell2, "Machine learning: Trends, perspectives, and prospects", Science,pp 1-7, 2015

[5] Pål Sundsøy1, Johannes Bjelland1, Asif M. Iqbal1, Alex "Sandy" Pentland2, and Yves-Alexandre de Montjoy, “Big Data-Driven Marketing: How Machine Learning Outperforms Marketers' GutFeeling",Springer, 2014

[6] "Artificial intelligence and machine learning in financial services", Financial Stability Board, 2017

[7] Georgios Paliouras, Vangelis Kakaletsis,"Machine learning and its application",pp 295-299, Springer,2001

[8]T.Thiraviyam, "Artificial intelligence marketing",

https://www.researchgate.net/publication/328580914_Artificial_intelligence_Marketing,2018

[9] Sahar F. Sabbeh,"Machine-Learning Techniques for Customer Retention: A Comparative Study", International Journal of Advanced Computer Science and Applications (IJACSA), 2018

[10] Dr. Amol Murgai,"Transforming Digital Marketing with Artificial Intelligence", International Journal of Latest Technology in Engineering, Management \& Applied Science (IJLTEMAS), 2018.

[11] Jim Sterne,"Artificial Intelligence for Marketing: Practical Applications", 2017

[12] Xiao Luo,Revanth Nadanasabapathy,A. Nur Zincir-Heywood,Keith Gallant,Janith Peduruge"Predictive Analysis on Tracking Emails for Targeted Marketing",Springer,2015

[13] Simon Kingsnorth, "Digital Marketing Strategy: an integrated approach to online marketing", Google Scholar, 2016

[14] Louis Columbus"10 Ways Machine Learning Is Revolutionizing Marketing”, Forbes, 2018

[15] Yogesh Hole,"Service marketing and quality strategies",Periodicals of Engineering and Natural Sciences, Vol 6,No 1, 2018 
[16] Srikanth Bethu,"Data Science: Identifying influencers in Social Networks", Periodicals of Engineering and Natural Sciences, Vol 6, No 1, 2018

[17] Aruna R Flarence,"Importance of Supervised Learning in Prediction Analysis", Periodicals of Engineering and Natural Sciences, Vol 6, No 1, 2018 